\title{
Crise et communication dans la sphère financière
}

\section{Robert Boublil}

\section{OpenEdition}

Journals

Édition électronique

URL : http://journals.openedition.org/communicationorganisation/2278

DOI : 10.4000/communicationorganisation.2278

ISSN : $1775-3546$

\section{Éditeur}

Presses universitaires de Bordeaux

\section{Édition imprimée}

Date de publication : 1 novembre 1999

ISSN : 1168-5549

\section{Référence électronique}

Robert Boublil, «Crise et communication dans la sphère financière », Communication et organisation [En ligne], 16| 1999, mis en ligne le 27 mars 2012, consulté le 19 avril 2019. URL : http:// journals.openedition.org/communicationorganisation/2278; DOI : 10.4000/ communicationorganisation.2278

Ce document a été généré automatiquement le 19 avril 2019.

(c) Presses universitaires de Bordeaux 


\title{
Crise et communication dans la sphère financière
}

\author{
Robert Boublil
}

\section{Introduction}

1 La crise financière qui est née et qui s'est résorbée en quelques semaines au 2esemestre 1998 appartient a un genre nouveau. D'abord, elle est restée principalement financière, et n'a pas contaminé fortement l'économie réelle, au moins en Occident. Elle a touché surtout les banques, et parmi elles, les plus grosses, au sujet desquelles on a invoqué le redoutable risque systémique (jeu de quilles). Elle a révélé le haut degré de financiarisation et de globalisation de l'économie. Elle s'est achevée enfin, non pas du fait d'une amélioration soudaine des fondamentaux (la Russie est toujours aussi insolvable, et l'endettement des pays asiatiques toujours aussi inquiétant aujourd'hui), mais sur le constat que les Autorités politiques et monétaires qui comptent dans le monde se sont remises dans leurs rôles respectifs, après une période de flottement, en effet préoccupant.

Dans cette crise, les acteurs des marchés financiers se sont plus intéressés aux autres acteurs qu'aux réalités tangibles qui, elles, n'ont pas fondamentalement changé. Ceci, sans doute parce que ces acteurs ont à résoudre une équation quasi-insoluble. Il leur faut : - comprendre instantanément une réalité économique de plus en plus complexe, et la traduire néanmoins dans des décisions simples, voire binaires (acheter ou vendre) ;

4 - imaginer la manière dont le reste du marché envisagera l'avenir, pour ne pas avoir à expliquer une décision prise à contre-marché ;

5 - continuer enfin d'enrichir les portefeuilles dont ils ont la charge, qui seront revus trimestriellement, et comparés à d'impitoyables indices.

6 On peut comprendre, dans ce type de circonstances, combien l'art de rassurer ou d'influencer ces acteurs, bref de communiquer, directement ou à travers certains média et prescripteurs, devient un enjeu important pour les émetteurs. 


\section{Autopsie d'une crise financière}

7 Juillet 1998. Le marché est de bonne humeur. L'indice Dow Jones poursuit son irrésistible ascension depuis 8 ans. La stratégiste Abby Joseph Cohen, la gourou des marchés Actions de la Banque Goldman Sachs à New York prédit qu'il franchira bientôt le seuil des 10000 points. Les valeurs du secteur bancaire européen connaissent une croissance tonique ( $+30 \%$ en 6 mois). La crise asiatique, qui avait fait l'objet de lourdes provisions en 1997, semble s'éloigner des préoccupations des opérateurs et gérants de portefeuilles, tous convaincus de l'imminence d'une vague de fusions et acquisitions que doit déclencher l'arrivée de la monnaie unique en Europe. Richard Grasso, Président du New York Stock Exchange, de passage à Paris pour la conférence de la Securities Traders Association, prononce l'oraison funèbre de la Debt Culture, et célèbre la renaissance d'une Equity Culture, désormais globalisée et fonctionnant en temps réel.

Les vances s'annoncent bonnes. N'était le sentiment, ici et la, que l'on n'avait pas assez acheté, que l'on aurait dû, que certains conseillers en gestion financière avaient manqué de punch, qu'ils auraient dû être bien plus bullish ${ }^{1}$ ! Triomphe de ceux qui ont gagné car ils avaient vu venir le coup. Cupidité insatisfaite de ceux qui ont hésité, ou trop attendu, et qui se trouvaient bien sots de n'avoir pas retiré leur épargne de précaution pour la reporter, tout entière, sur les actions.

Pourtant, tout n'est pas clair en et été 98 . La situation des 5 tigres $^{2}$ se révèle sous un jour de plus en plus alarmant : crise de liquidité et vacance de pouvoir en Thaïlande ; émeutes et instabilité politique en Indonésie; révélation des vrais niveaux d'endettement des Chaebols ${ }^{3}$ de Corée.

10 Au Japon, la Diète continue de s'opposer à un plan de sauvetage du système bancaire, devenu presque globalement insolvable au regard de la réglementation prudentielle bancaire mondiale 4 , qui finira par coûter à l'État un effort de recapitalisation équivalent de $12 \%$ du Produit Intérieur Brut (PIB) japonais (!). A Hong Kong, des doutes commencent à poindre sur la capacité de l'Autorité Monétaire du Territoire à maintenir le peg ${ }^{5}$ face à la pression des spéculateurs. En Russie, Boris Eltsine qui a démis Victor Tchernomyrdine, compte sur un jeune Premier Ministre sans expérience nommé à sa place, Serguei Kirienko, pour qu'il s'attaque sérieusement aux problèmes économiques du pays. Au Brésil, une élection présidentielle est attendue pour la fin de l'été, laissant l'État sans direction effective pendant la campagne. Aux États-Unis enfin, le Monicagate continue d'empoisonner la vie publique. Le scandale accrédite chaque jour un peu plus l'idée que Bill Clinton pourrait être destitué, et que de toute façon, il a perdu une grande partie de sa crédibilité. Mais il souligne surtout à quel point les parlementaires américains manquaient de vision, alors même que se préparait à éclore la crise financière la plus grave depuis 1929 .

11 Soudain en effet, le 17 août, la Russie fait défaut, pour la seconde fois au cours du même siècle. La crise de 1998 éclate alors. Depuis la mi-août jusqu'à la mi-octobre, l'affolement gagne les marchés de capitaux, et atteint même les responsables les plus sereins dans les grandes banques. Les réunions d'analystes organisées, comme chaque année en cette saison, pour commenter les résultats du premier semestre, tournent à la débandade. Des banques aussi peu exposées aux risques émergents ${ }^{6}$ que le Crédit Commercial de France perdent jusqu'à $10 \%$ en une seule séance, au seul motif que la banque a une filiale au 
Brésil. La dernière semaine de septembre est dévastatrice : les actions de la plupart des grandes banques françaises et européennes abandonnent $10 \%$ par jour en bourse. Des rumeurs insistantes parient de faillite imminente pour Lehman Brothers, Paribas, la Société Générale, et bien d'autres. On verra, au début 99, à l'examen des résultats annuels, qu'il s'agissait d'exagérations. En quelques jours en Septembre, les marchés de capitaux se polarisent selon une stratégie moutonnière de " flight to quality ${ }^{7}$, faisant grimper les spreads ${ }^{8}$ des émetteurs d'obligations n'appartenant pas aux toutes premières catégories (États Américain, Allemand ou Français), et renchérissant le coût de la ressource des acteurs privés de l'économie au point de créer un véritable "Credit Crunch ${ }^{9}$. A l'inverse, les obligations d'États incontestables, devenues le refuge unique des investisseurs, voyaient leur cours grimper, et leur rendement diminuer d'autant.

Le 25 septembre, la révélation de la faillite de LTCM (Long Term Capital Management) constitue le point d'orgue de la crise : on découvre la véritable nature des Hedge Funds ${ }^{10}$; et qu'un seul d'entre eux, LTCM, dirigé entre autres par deux prix Nobel d'économie, menace la stabilité des finances publiques américaines elles-mêmes. C'est alors qu'intervient Alan Greenspan, Gouverneur de la Réserve Fédérale américaine, pour régler le problème posé par LTCM. Il oblige - par la persuasion, vue l'absence de réglementation - un tour de table incluant quatorze des plus prestigieuses banques de la planète, à reconstituer les fonds propres de LTCM, pour permettre le débouclement dans le calme et sur la durée, des contrats souscrits par ce Hedge Fund auprès des banques. Car s'il avait fallu exécuter les gages donnés par LTCM à ses contreparties, une grande partie de l'encours de Bons du Trésor Américains aurait été déversée brutalement sur le marché, faisant baisser leur cours, et partant, augmenter les taux d'intérêts du dollar.

13 L'inversion du processus de crise se joue à ce moment la, alors que pendant les six semaines qui ont précédé l'épisode LTCM, aucune autorité ne semblait présente sur la scène. Ainsi, qu'il s'agisse du FMI, trompé par les Russes et attendant le vote américain pour toucher 18 milliards de dollars de financement; de l'Autorité Monétaire de Hong Kong, attaquée par les spéculateurs pour renoncer au peg; du gouvernement japonais, incapable d'imposer un plan pour son système bancaire ; du gouvernement brésilien, non encore en place ; du Président américain, attendant son éventuelle destitution; ou enfin du pouvoir russe, fragile et peu fiable: aucune autorité ne se trouvait en place pour résister aux forces du marché.

14 Aucune sauf précisément Alan Greenspan. C'est lui qui par un geste fort, consistant à réunir une vingtaine de grandes banques mondiales pour les obliger à secourir LTCM, a restauré la confiance des marchés. Cet épisode a été suivi de peu par un débouclement du problème du financement du FMI par le Parlement américain. Les marchés ont alors perçu qu' « il y avait un pilote dans l'avion », et sur la foi de ce seul sentiment, ils ont inverse leur attitude. Inutile de rappeler que le risque russe ne s'est, lui, pas amélioré d'un iota, que la situation de la plupart des marchés émergents (en Asie comme en Amérique latine) devrait mettre longtemps à s'inverser. C'est donc sur l'observation d'attitudes plus que de faits concrets que les acteurs des marchés financiers ont retrouvé le sourire, et partant, mis fin à une crise potentiellement catastrophique. 


\section{Financiarisation, globalisation, et complexité croissante de l'économie}

15 Il n'est pas contestable qu'en cinquante ans, les progrès des technologies de l'information et de la communication ont fait subir à l'économie mondiale une mutation au moins égale en importance à ce que fut la révolution industrielle. Le secteur financier, grand utilisateur de ces technologies, était ainsi prédisposé à une grande mutation.

Or cette période démarre en même temps que la signature des accords de Bretton Woods, qui ont tenu pendant 27 ans avant d'être abrogés en 1971 par Richard Nixon. Ces accords étaient d'essence économique, bien qu'ayant reposé principalement sur des régies financières. Leur objectif était de permettre à l'économie mondiale de fonctionner dans un environnement aussi stable que possible, caractérisé par la fixité du rapport de change entre les monnaies. Celle-ci permettait aux acteurs de l'économie de prendre leurs décisions sur la base des seules deux variables cardinales du commerce: la marge bénéficiaire (prix de vente moins prix de revient) pour les entreprises ; le rendement des capitaux employés pour les investisseurs. Le risque de change existait, certes, du fait de l'éventualité de dévaluations pouvant être décidées ici ou la, mais ce risque pouvait être facilement couvert auprès des banques, aux termes de calculs assez simples intégrant les probabilités de survenance de tels événements. Quant aux investisseurs, il leur était plus facile, avant 1971, d'analyser la pertinence d'un investissement : les taux d'intérêts a long terme étant a l'époque fixes, on pouvait connaitre précisément l'écart de rentabilité attendue de l'investissement, et le coût de la ressource venant le financer. Cet écart était censé absorber, entre autres, le risque de dévaluation en cas de mismatch (actif et ressources correspondantes exprimées en deux monnaies différentes).

La décision de Richard Nixon a brutalement multiplié les variables, et donc des incertitudes. En schématisant, trois paramètres antérieurement à peu prés stables, devenaient du même coup variables : le coût de l'énergie, du fait de l'aléa sur la valeur du Dollar américain, a entrainé une incertitude sur les prix de revient ; la valeur relative des monnaies entre elles ne permettait plus aux exportateurs de connaître leurs revenus avant de les avoir effectivement encaissés; enfin les taux d'intérêts, prenant en compte dorénavant la couverture du facteur inflation, devinrent un autre paramètre essentiellement mobile, en ce qui relevait tant du calcul des prix de revient des entreprises (poids des frais financiers), que de celui du rendement net pour les investisseurs.

18 Si l'on ajoute à ce triplé, le facteur de prolifération des liquidités globales, nées des crises du pétrole consécutives, on peut mieux comprendre l'importance croissante des prestataires de services financiers dans l'économie :

19 - nécessité de trouver des solutions pour immuniser la marge commerciale des entreprises exportatrices contre le risque de change ;

20 - nécessité d'immuniser les agents économiques (entreprises et épargnants/ investisseurs) contre le risque de taux ;

21 - nécessité d'adapter les circuits et marchés mondiaux pour fournir la meilleure liquidité possible aux détenteurs de capitaux, et ce à l'échelon global.

22 Ces nouveaux besoins ont accru l'importance du rôle des prestataires financiers dans le monde, non plus seulement comme fournisseurs de ressources, mais désormais comme 
conseils ou gestionnaires, puis contreparties, dans ces nouveaux métiers. Ce passage brutal à une économie de plus en plus financière a constitué l'acte de naissance effectif de l'économie globale de marché - comme nous allons le voir - et consacré la prééminence du financier par rapport à l'économie réelle. Dans cette nouvelle donne, trois domaines particuliers ont explosé et modifié le paradigme de l'industrie financière.

\section{Dérivés de change}

La première innovation financière majeure date de la fin de la décennie 70 et revient aux banques d'investissement new-yorkaises. Baignant dans une culture de courtage, elles imaginèrent de mettre face à face, dans un même contrat de SWAP $P^{11}$, deux agents économiques ayant une "position", (c'est à dire un solde) dans une monnaie donnée, jugée indésirable, par chacun d'entre eux. Si la position des deux contreparties est de même montant, de même durée et opposée, et si elle est jugée par chacun d'entre eux potentiellement perdante, on peut comprendre qu'ils envisagent de l'échanger, et ainsi de couvrir leur risque. Ils conviennent alors de faire un pari, au dénouement duquel on constate qui a gagné et qui a perdu ${ }^{12}$. L'opération, qui porte sur une anticipation d'évolution d'une différence (et non des valeurs principales), mérite ainsi son nom de « dérivé ».

\section{Dérivés de taux}

La même idée consistant à parier sur une évolution a bientôt été appliquée au marché des taux. L'échange des revenus d'une créance à taux fixe contre ceux d'une créance à taux variable pouvait convenir à des investisseurs en fonction de l'emploi qu'ils avaient, à un moment donné, de ces ressources. Lorsqu'un Swap de taux se dénoue, le perdant paie au gagnant le différentiel d'intérêts, que l'on calcule a posteriori, une fois connu l'écart de taux qui a couru pendant la période du pari. Ce type de produit dérivé a fait florès, avec la croissance des marchés de taux. Ce faisant, on peut mesurer combien il relève du pari anticipatif, de la part des acteurs qui s'y livrent.

Qu'il s'agisse de swaps de taux ou de swaps de monnaies, la finalité de ces activités est essentiellement de couvrir un risque pour un agent de l'économie réelle. Aussi l'existence de spéculateurs purs (c'est a dire autre que les contreparties primaires) est-elle salutaire. Mais il convient de noter que les Banques se sont mises rapidement à se livrer, elles mêmes, à une activité pour compte propre, et dans le cas où les marchés évoluent dans un sens contraire à leur anticipation, ce sont elles qui enregistrent directement des pertes à caractère spéculatif.

\section{Dérivés Actions}

Dans le prolongement de la même idée d'échanger des risques sur positions de change ou de taux, on inventa les dérivés-actions. Mais a la différence des deux autres marchés, le marché des actions ne fait intervenir que des détenteurs d'actifs, qui sont par construction confrontés au risque de cours, à la différence des entreprises qui ont le devoir de s'en protéger.

Ainsi partant d'une diversification de leur métier qui a pour finalité la couverture des risques des acteurs de l'économie réelle, les Banques ont évolué vers une activité de 
marché, largement sinon totalement tournée vers le pari spéculatif, dont la particularité est qu'il suffit de trouver une contrepartie pour réaliser une affaire. Ces marchés purement financiers parviennent a s'autoalimenter, en se déconnectant petit à petit, des besoins de l'économie réelle. L'aboutissement paradoxal de ce nouveau paradigme est que les banques peuvent gagner - sauf en cas de krash des marchés - beaucoup plus qu'en prêtant des fonds à leurs clients, ce qui est pourtant leur finalité première.

\section{Les marchés émergents}

Les banques n'ont toutefois pas renonce à leur métier de prêteur. Mais dans ces activités traditionnelles il leur est apparu que les marges bénéficiaires sur capitaux employés devenaient trop minces sur leurs marchés domestiques. Vu les taux de croissance des économies émergentes, elles décidèrent (toutes ensemble, comme c'est souvent le cas dans l'industrie bancaire) de s'y engager. Elles ont suivi en cela l'irréversible mouvement de globalisation de l'économie. En prenant sans doute les précautions voulues pour intégrer dans leur "pricing» l'élément de risque additionnel qui existe sur les marchés moins mûrs, donc moins fiables, les banques appliquèrent leurs techniques modernisées à ces économies naissantes. Ce qui ne fut pas escompté, c'est l'effet domino qui s'est produit sur la quasi totalité de ces marchés, quel que soit le continent. Et pourtant, le surendettement de la Thaïlande a-t-il à voir avec l'économie brésilienne? La question peut être posée non pas en termes économiques (ou alors trés indirectement), mais plutôt en termes "d'assimilation ", par les marchés, de situations pouvant entrer dans la même catégorie. Il s'agit ici de l'effet sémantique du mot « émergent » dont la conséquence est celle d'un déplacement des capitaux vers des catégories moins dangereuses. Ceci, notons le, n'a été possible que parce que les banques savent aujourd'hui, déplacer lesdits capitaux à la vitesse de la lumière.

L'exemple qui précède illustre que la globalisation, bien qu'ayant été promue et stimulée par les grands acteurs financiers, ne les a toutefois pas épargnés, ce qui évoque qu'il s'agit une créature qui échappe à son maître, comme dans le mythe.

La financiarisation croissante et la globalisation ont créé ensemble un univers nouveau, beaucoup plus complexe et vaste, et dans lequel les facteurs de risque sont mal maîtrisés par ceux-là mêmes qui sont censés pouvoir les dominer. Dans et univers, les processus usuels d'analyse s'avèrent insuffisants, voire défaillants. Ils laissent les marchés sur leur faim et désorientés. Lis perdent toute légitimité lorsqu'une crise surgit, comme cela a été le cas en 1998.

31 Les autorités de contrôle des marchés et de la profession bancaire se sont d'ailleurs alarmées de cette évolution: en décembre 1998, une publication cosignée par Michel Prada, Président de la Commission des Opérations de Bourse (COB) et Jean Claude Trichet, Gouverneur de la Banque de France, intitulée La Transparence Financière ${ }^{13}$, constate que :

"les événements récents qui ont secoué les systèmes financiers asiatiques illustrent parfaitement... le danger d'une information opaque et insuffisante ".

et note en particulier que :

"les années récentes ont montré de manière édifiante que des entreprises pouvaient délibérément adapter une stratégie spéculative peu compatible avec leur objet social... sans disposer des instruments de mesure el de contrôle des positions qu'elles prenaient sur des marchés extrêmement volatils". 
Dans ce contexte, les auteurs mettent "les spécialistes de l'information face à leurs responsabilités", ce qui semble être leur principal objectif, conscients qu'ils sont des risques liés a la pratique d'une communication mal normée, et reconnaissant que les informations factuelles ne suffisent plus, l'anticipation devenant un élément à part entière des messages aux marchés, soit directement soit par les divers médiateurs qui y ont leur place :

"les analystes financiers jouent un rôle clef en communiquant au public une appréciation sur l'évolution des perspectives des sociétés, a partir de l'information financière, qu'elle s'appuie sur l'examen des comptes financiers ou sur l'extrapolation du parcours boursier de la valeur... Comme pour les analystes financiers, la qualité de l'anticipation est un élément crucial de la crédibilité des agences de notation ».

\section{Information et communication financière en temps de crise}

La finance dérive, en principe, des mathématiques, en prenant comme point de départ la comptabilité. Mais le domaine a connu une formidable explosion, notamment depuis l'invention des tableurs (Excel...). Les marchés financiers, quant à eux, sont, par construction, le lieu de rencontre d'acteurs effectuant des opérations symétriques, à un moment donné. La psychologie a toute sa place dans cette confrontation, car les acteurs vont jouer chacun à prédire l'avenir, tout autant que la réaction de leurs contreparties et des marchés. Les méthodes graphiques ou quantitatives, prédisent le comportement des marchés en fonction d'historiques reflétant les attitudes collectives. Ces récurrences sont prises en compte pour bâtir des modèles permettant de quantifier la perte potentielle d'une position, dans la situation défavorable considérée comme extrême. De tels modèles, bien que très élaborés, ont été défaillants au cours de la crise de 98 , ce qui démontre qu'une crise financière est, par hypothèse, imprévisible par le calcul, le cas extrême pouvant toujours être dépassé. En revanche la survenue prochaine d'une crise peut être invoquée au terme d'un raisonnement de bon sens, voire sur une simple intuition. Ce type de pronostic se rencontre fréquemment de la part d'analystes, d'économistes, et même quelquefois - ce qui est encore plus fortement pris en compte par les marchés - par les gouverneurs des banques centrales eux-mêmes, dans un souci de refroidir un peu l'enthousiasme haussier spéculatif des marchés.

La communication financière met en présence trois catégories d'acteurs: les émetteurs (entreprises émettrices de valeurs mobilières); les récepteurs (acteurs du marché de capitaux: investisseurs et traders) et enfin les médiateurs (journalistes, analystes financiers, économistes et agences de notation). Dans la configuration actuelle des marchés, et en temps de crise en particulier, les trois catégories joueront leur partition selon une grille, dont nous verrons qu'elle peut être désormais très largement subjective, ce qui explique en particulier la préoccupation des autorités chargées de veiller au bon fonctionnement des marchés.

\section{Émetteurs et Récepteurs}

Les émetteurs sont statutairement astreints à respecter des obligations réglementaires en matière d'informations communiquées au marché. Ces régies sont essentiellement d'ordre comptable et déontologique. 
la complexité de l'économie moderne globale, la sophistication des instruments financiers, la puissance des moteurs de calcul informatiques, l'intelligence des mathématiciens qui développent les modèles, la difficulté qu'il y a à contrôler ces modèles, le mode de rémunération des gens de marchés (encouragés à augmenter les flux d'affaires, sans considération pour le niveau de risque), la vitesse d'exécution des transactions, sont autant de facteurs qui rendent le système d'information financière réglementaire presque totalement caduc et inadapté. Les acteurs du marché vont donc, pour la plupart, s'intéresser à d'autres modalités d'appréhension du réel. Et c'est pourquoi ils sont les principales cibles des stratégies de communication, menée par les émetteurs, dont le but de guerre est de s'attirer leur sympathie. Ce n'est pas par hasard que le vocabulaire employé dans la communication financière moderne évolue dans un sens compatible avec cette stratégie. On parle ainsi de plus en plus de «Economie Value Added» ou de "Valeur pour l'actionnaire » ou encore de "Création de Valeur». Ces expressions ne ressortissent plus du lexique comptable ou financier traditionnel (ex: Bénéfice par action; ou ratio Prix/ Bénéfice), mais semblent appartenir plutôt à la sémantique des idéologues, et même en poussant un peu, de la religion.

Quant aux récepteurs, les acteurs directs des marchés, il convient de rappeler d'abord qui ils sont, et ce qu'on leur demande. Il s'agit d'investisseurs, gérant directement leurs capitaux (assureurs par exemple, ou fonds de pension), ou de gestionnaires mandatés par les détenteurs ultimes (gérants de fonds de placements collectifs ou gérants de fortunes privées). On trouve dans les marchés également, mais c'est une tout autre catégorie et une autre culture, des «traders » qui procèdent à des transactions dont le dénouement est prévu a très court terme, et qui interviennent, en principe, à l'intérieur d'un plafond de capitaux maximal autorisé par leur règlement et/ou leur hiérarchie, pour « faire leurs jeux ».

39 Cet ensemble hétérogène d'acteurs se nourrit d'informations brutes (écrans des agences de presse telles que Reuters ou Bloomberg par exemple, qui transmettent en temps réel les nouvelles du monde et des entreprises), ou digérées (publications d'économistes ou d'analystes financiers). Les émetteurs se livrent régulièrement à une fourniture d'informations, qui relève de la communication. Admettant que l'information réglementaire est insuffisante, ils émettent des messages non nécessairement vérifiables, en soignant puissamment la convivialité, l'accessibilité, la facilité de compréhension, ou de comparaison. Ils se livrent à un véritable « marketing ", qui ne dit pas son nom, car la tenue pseudo-scientifique de cette communication la, reste de mise. On va ainsi présenter l'information sous son jour le meilleur, en choisissant son terrain (réunion, ou communiqué de presse, ou support CDRom etc.) ainsi que le moment approprié (être le premier à sortir ses résultats semestriels, par exemple, ou attendre que le concurrent le fasse d'abord...). Tout sera matière à envoyer le bon message au bon moment. Le contenu manipulatif de ce procédé est patent. Surtout si l'on rappelle que les données factuelles deviennent non seulement trés complexes à comprendre, même pour des gens trés qualifiés, mais que, de plus, elles peuvent devenir caduques du jour au lendemain, car une position financière peut s'inverser plusieurs fois par jour.

40 Ajoutons que la mission des acteurs du marché n'est plus de tout repos. Il existe désormais des indices auxquels on peut se mesurer à tout moment. Le culte de la performance règne en maître dans le domaine des actions, où pourtant il est connu que les cycles sont les plus longs. Or les performances de gestion sont actuellement mesurées trimestriellement. Il est demandé aux gérants, enfin, de pouvoir justifier de leurs 
stratégies en termes simples, alors même que la complexité est à son maximum. N'importe qui peut avoir accès, à tout moment au "Consensus ", c'est a à dire aux prévisions de bénéfices de l'ensemble des analystes sur une valeur donnée. Investir dans un sens opposé au consensus peut relever parfois de la bravoure. Il n'est même plus permis de patienter un peu avant d'investir les capitaux, vu le très faible niveau des taux d'intérêt actuels, et les très fortes performances, sur cycles courts, de la bourse.

Ces conditions forment le terreau idoine pour que la communication s'impose comme le moyen synthétique, pour un émetteur, de s'attirer la sympathie des marchés. On combinera les images, les chiffres, les mots, les discours, et parfois même les silences qui en disent long, pour atteindre ce résultat. Le coefficient personnel des dirigeants (charme, autorité) sera pris en compte pour se faire une opinion. En temps de crise, la nature de l'information (bonne ou mauvaise) sera mise au second plan, ainsi que démontre l'étude réalisée par la $\mathrm{COB}$ et la Commissions Bancaire (op. cit., note $\mathrm{n}^{\circ} 13$ ) :

"La communication de crise, même lorsqu'il s'agit en apparence de mauvaises nouvelles, entraine le plus souvent des réactions favorables du marché, qui semble récompenser la transparence ".

La même étude évoque le comportement des banques peu de temps après l'éclosion de la crise asiatique, en janvier 1998 :

"s'agissant de l'événement le plus récent, (la crise asiatique), les établissements ont adopté une attitude commune visant a rassurer: les grandes banques ont en effet eu à coeur de la replacer en perspective, publiant dès janvier 1998 des prévisions plutôt optimistes du résultat annuel 1997 ».

43 Cette citation est particulièrement éloquente pour mesurer les risques de biais qu'induisent les stratégies de communication en temps de crise: les banques ne connaissaient pas, en janvier 1998, l'ampleur de la crise asiatique. La preuve, c'est qu'elles ont dû compléter leurs provisions au cours de l'exercice 1998, en décidant de couvrir ce risque à hauteur de 25 a $30 \%$ environ du montant de leurs engagements, alors qu'il ne l'était que de $10 \%$ à $15 \%$ au moment de la clôture des comptes de 1997. Elles auraient donc eu tort de vouloir rassurer sur la crise asiatique. En revanche, ce qu'elles ont souhaité probablement faire, c'était rassurer sur l'impact relativement «tolérable » de cette crise sur leurs comptes, car par ailleurs, elles ont réalisé de bonnes affaires sur certains marchés, ce qui a pu compenser leurs provisions sur l'Asie.

On voit, par les exemples qui précédent, que la perspicacité des analystes et des investisseurs devient tout aussi importante que leur compétence technique. Au plan psychologique, notamment, car il leur faut désormais faire le tri entre ce qui relève de la réalité et ce qui procédé des stratégies de communication.

\section{Médiateurs (journalistes, analystes financiers)}

45 A l'instar des situations militaires, où les correspondants s'intéressent plus à détecter le bluff, à estimer le moral des protagonistes, ou à faire le tri entre information et désinformation, les acteurs des marchés financiers ont de plus en plus la même attitude : chercher le sens caché des choses; évaluer s'il y a eu dissimulation, ou encore tester la cohésion entre les membres de l'équipe dirigeante. C'est sur ce type de données que les marchés bougent. Le titre Alcatel, par exemple, représentant l'une des valeurs boursières les plus importantes en Europe, a perdu $40 \%$ en une seule séance à la bourse de Paris, sur 
un sentiment de flottement perçu par les participants à la réunion où étaient présentés les comptes du 1er semestre 1998 !

Dans ce type de réunions (tenues deux fois par an en principe) tout se joue sur le non-dit, ou encore sur l'«après-dit ", c'est à dire les discussions informelles tenues entre managers et analystes, ou encore entre analystes eux-mêmes, visant à s'échanger des impressions (dans le meilleur des cas), ou à distiller des informations prétendues confidentielles, pour modifier un sentiment (dans le pire des cas). Dans ce jeu-là, bien des coups sont permis, car nul n'aura à démontrer son point par des faits vérifiables. Les sous-entendus, les sourires appuyés, les « suivez mon regard » sont langage courant. Ces réunions ressemblent aussi à des grand-messes, servies par des pontes dont on aimerait connaître l'état de santé réel, et non celui allégué. Les ouailles respecteront le déroulement liturgique, mais iront de leurs commentaires sur une toux sèche par-ci, un tremblement dans la voix par-là, entendus pendant l'office. Les plus imaginatifs évoqueront, à la sortie, un ragot colporté au énième degré par une de leurs relations, soi disant bien au courant du dossier médical de l'intéressé. L'activité d'influence de l'autre, mais aussi d'auto-persuasion, existe dans cette sphère où la fiabilité, la transparence et la loyauté sont pour le moins imparfaites.

Mais les médiateurs que sont les journalistes et les analystes financiers sont aussi l'objet d'influences tant de la part des émetteurs - ce qui est facile à comprendre - que de celle des investisseurs, qui peuvent, par leur inquiétude par exemple ou pour d'autres motifs, faire pression sur le jugement des analystes.

Pendant la crise de l'été 1998, j'ai suivi, comme analyste du secteur bancaire, avec beaucoup de tension, la situation des banques prises dans la tourmente. On peut imaginer la pression qu'ont exercé sur moi les investisseurs auxquels j'avais précédemment conseillé l'achat de tel ou tel titre, et qui se trouvaient pris, à ce moment la, dans une dégringolade jamais vue (division par 2 du cours entre juillet et octobre 1998 - mais il serait injuste d'oublier que les cours avaient doublé au premier semestre). Le nombre de visites que j'ai eues, ou d'appels que j'ai reçus, à cette époque la, de la part de personnes ayant supposément entendu des rumeurs sur telle ou telle banque, est inimaginable. Ces démarches auraient pu avoir un effet dévastateur sur ma sérénité, si je n'avais pas blinde mes capteurs. J'exigeais par exemple que l'on me cite nommément la source de la rumeur auprès de ceux qui la colportaient, pour que j'accepte de la considérer dans mon analyse. Je fermais littéralement la porte de mon bureau pour me protéger de ces assaillants paniqués et potentiellement contagieux. Au milieu de cette tourmente, je publiais le 5 octobre, une étude dont je crois bon de citer ici un extrait de la page de résumé et conclusions :

"Le segment des valeurs bancaires françaises a subi un retournement à $180^{\circ}$ en quelques mois, passant d'un sentiment de "Beau Fixe » boursier à un " Avis de tempête ». L'amplitude relève plus de la crainte panique d'un effondrement du système bancaire mondial touchant les plus grandes institutions, que d'une analyse des risques individualisés de chaque établissement. L'égalité dans la chute des cours traduit une totale absence de différenciation. Le pronostic sur les chances de règlement de la crise actuelle est aléatoire, car tout dépendra du sang froid des décideurs. Toutefois, nous reconnaissons dans des cas récents, une prise en main énergique des autorités de contrôle concernées (aux USA, au Japon et à Hong Kong), visant à ne pas laisser la spéculation l'emporter, à réduire, au besoin par la coercition, l'inquiétante volatilité des marchés, et à faire redémarrer les économies sinistrées. Dans l'hypothèse où ces politiques parviennent a calmer le jeu, on reviendra a une analyse plus sereine et fondamentale des valeurs concernées, et on pourra évaluer le risque relatif de 
chaque banque par rapport aux 5 « bulles « de 1998, 3 d'entre elles ayant déjà explosé, et les 2

dernières étant encore latentes... ». de me placer vis à vis de mes lecteurs: il s'agissait d'abord pour moi, non pas de démontrer pourquoi la crise allait se résorber (j'en aurais été bien incapable), mais plutôt dans quelles conditions elle pourrait l'être. Ces conditions relevaient uniquement de la psychologie des acteurs et non des données économiques factuelles. J'informais mes lecteurs sur la nature de mon propre pari, en soulignant qu'il s'agissait bien d'un pari. Puis me plaçant dans un scénario de sortie de crise, je formulais mes recommandations. Ceux de mes lecteurs qui ont adhéré à ma démarche et procédé au même pari que moi s'en réjouissent aujourd'hui, la bourse s'étant reprise de façon spectaculaire depuis la crise. Mais ce qui a été dit explicitement dans ce texte relève de l'anticipation, dont la COB et la Commission Bancaire elles-mêmes disent qu'elle incombe à l'analyste.

\section{Conclusion}

Nous avons rappelé les circonstances de la crise financière de 1998, de sa survenue et de sa résorption.

Nous avons pu montrer combien la complexité croissante de l'économie, au cours des 50 dernières années, sa globalisation et sa financiarisation, ont contribué à créer un nouveau paradigme en ce qui concerne les relations entre les différents acteurs.

Ces considérations nous conduit à voir que dans ce nouvel univers, le pronostic et l'anticipation constituent des activités clefs. A fortiori, en temps de crise, on peut bien comprendre que les émetteurs (seuls détenteurs de l'information réelle), sont soucieux d'éviter d'être les victimes de telles crises. Ils feront donc tout ce qu'ils peuvent pour influencer le pronostic dans le sens le plus favorable. Dépassant l'état de simples fournisseurs d'information, ces émetteurs se livreront à de la communication, comme stratégie et moyen d'agir sur le marché, et de fait d'influencer le réel.

\section{NOTES}

1. De Bull (Angl.) : Taureau. Sens figure : haussier; anticipant une hausse des cours. Inverse de Bearish (Bear : Ours).

2. Corée du Sud, Thaïlande, Philippines, Malaisie et Indonésie.

3. Conglomérats mixtes, industriels et financiers, typiques de la structure d'entreprise sud coréenne.

4. Une réglementation a été élaborée, applicable à l'ensemble de la profession bancaire dans le monde, par les Accords de Bâle. Son application est contrôlée par la Banque des Règlements Internationaux (BRI). Elle pose que le capital d'une banque ne saurait être inférieure à un certain ratio (complexe), au dessous duquel la banque concernée est contrainte d'augmenter son capital, sous peine de perdre sa licence.

Communication et organisation, $16 \mid 2012$ 
5. lien de parité fixe, convenu entre les Autorités monétaires des deux pays concernés.

6. Risques (crédits, garanties données, titres de placement ou d'investissement) contractés sur des pays dont l'économie émerge (Asie, Amérique latine, et plus récemment Europe de l'Est) et qui connaissent de ce fait des taux de croissance très élevés.

7. Fuite vers la qualité : recherche de titres de créances sur lesquels le risque est considéré comme très faible ou nul.

8. Différence entre le rendement des obligations d'un émetteur privé ou semi-public et le taux de rendement des obligations d'État dans la même monnaie.

9. Pénurie de Crédit, consécutive à la frilosité croissante des prêteurs.

10. Littéralement «Fonds de couverture ». En réalité il s'agit de fonds spécialisés, généralement domicilies aux Bermudes, et qui se livrent à une activité de contrepartiste sur les marchés de capitaux, avec un très important effet de levier (ratio entre les capitaux propres et le total des engagements). Cette activité quasi-bancaire échappe ainsi à la réglementation prudentielle (voir supra note 4). Les Hedge Funds sont les principaux intervenants spéculatifs (en dehors des banques elles-mêmes). Le célèbre financier Georges Soros, gère quelques grands Hedge Funds.

11. Échange, en Anglais.

12. Le principe est le suivant : l'entreprise française A détient une créance de 1000 \$ qui échoit dans 3 mois, et craint une évolution défavorable du cours du change $F F / \$$ à cet horizon. Inversement, l'entreprise américaine B détient une créance de $5600 \mathrm{~F}$. qui échoit dans 3 mois qu'elle souhaite voir exprimer en $\$$ dans ses comptes. A et $B$ swappent leurs droits à créance, et a l'échéance B recevra 1000 \$ et A recevra 5600 F. L'échange peut s'accompagner d'un paiement a l'origine par l'une des deux parties a l'autre, reflétant le pronostic des marchés sur l'évolution attendue du cours des changes. Au bout des 3 mois A et B pourront constater si le cours $\mathrm{FF} / \$$ est différent de 5,60 (parité à la date où pari a été fait) et vérifier si elles ont gagné ou perdu en s'engageant dans ce pari. Mais en tout état de cause, elles auront pu réaliser leur opération commerciale en uniformisant la monnaie dans laquelle s'expriment à la fois leurs revenus et leurs coûts.

13. Publié par la Banque de France $4^{\mathrm{e}}$ trimestre 1998.

\section{RÉSUMÉS}

La crise financière de 1998 et sa sortie, ont surtout été affaire de communication.

La complexité induite par la globalisation, oblige les acteurs des marchés à renoncer a comprendre la réalité des problèmes. Ils ne font plus que parier sur les capacités des décideurs, et pressentir l'orientation des marchés. Ils se fondent sur des sentiments plus que sur des faits, et ce, avec bonne conscience, car la visibilité est nulle.

L'analyse des faits cédant la place à l'interprétation et au pari sur le sens du consensus, les avis des gourous seront prisés.

Dés lors, la communication peut devenir, en soi, la source du déclenchement ou de la résolution des crises. Et les émetteurs, acteurs de l'économie réelle, se mettront à gérer la communication presque plus que leurs entreprises.

The 1998 crisis, and its resolution have largely been a matter of communication. 
The complexity genarated by globalisation forces the markets to forego the comprehension of reality. They merely take a bet as to managements' capabilites, and markets'orientations. They base their decisions more on sentiments than facts, and with good conscience as visibility is nil.

The analysis of facts yields in front of interpretation and sentiment as to how the consensus might shape up. gurus'views become praised

In this context, communication can become, per se, a triggering factor for crises, and also for the resolution thereof. And in turn managers, the decision makers of the real economy, do manage communication, as much, if not more, than their own companies.

\section{AUTEUR}

\section{ROBERT BOUBLIL}

Robert Boublil est né en 1945. Diplôme d'études supérieures en administration des entreprises et en finance, il a exercé la plus grande partie de sa carrière dans l'industrie financière. Auprès de la Bank of Nova Scotia à Toronto. Puis à Paris comme cadre dirigeant de la filiale française de Barclays Bank, dont il a été le Directeur Général Adjoint, après avoir occupé diverses fonctions opérationnelles et fonctionnelles. Depuis 1994, Robert Boublil est analyste-consultant auprès de la Société de Bourse Wargny, à Paris, spécialiste des valeurs bancaires et d'assurance en Europe. 